\title{
RURALIDAD, CUIDADOS Y POLÍTICAS PÚBLICAS REFLEXIONES A PARTIR DEL CASO DE URUGUAY
}

Paola Mascheroni Laport

\section{Resumen}

Uruguay es un país pionero en políticas que buscan desfamiliarizar y desfeminizar el cuidado, pero su desarrollo ha estado centrado casi exclusivamente en contextos urbanos y las experiencias de cuidados en el medio rural aún son muy acotadas. El propósito de este artículo es avanzar en el conocimiento sobre las políticas públicas del cuidado implementadas en la última década en la ruralidad uruguaya. Se muestran las principales limitantes para su implementación en los territorios rurales, las que están vinculadas a factores estructurales y culturales. Se concluye que es necesario tratar simultáneamente estos factores para impulsar transformaciones tendientes a promover la corresponsabilidad social y de género en el cuidado.

Palabras clave: cuidados, hogares rurales, políticas públicas.

\section{Abstract}

Rurality, care and public policies. Reflections from the case of Uruguay

Uruguay is a pioneer country in policies that seek to de-familiarize and de-feminize care, but its development has been focused almost exclusively on urban contexts, and care experiences in rural areas are still very limited. This article describes and analyzes the care policy in rural areas within the created National System of Care of Uruguay. This article argues that because the main limitations are linked to structural and cultural factors, it is necessary to address these factors simultaneously in order to promote social and gender co-responsibility

Keywords: care, rural households, public policies.

Paola Mascheroni Laport: Doctora en Sociología. Docente e investigadora del Departamento de Sociología de la Facultad de Ciencias Sociales, Universidad de la República, Uruguay.

ORCID iD: 0000-0001-7244-2690

Email: paola.mascheroni@cienciassociales.edu.uy 


\section{Introducción}

En las últimas décadas ha surgido una literatura creciente sobre cuidados desde una perspectiva de género, pero aún son muy escasos los trabajos que se centran específicamente en los espacios rurales y sus particularidades.

El término cuidado, si bien es un concepto en continuo proceso de (re) construcción teórica, implica en sentido general la producción de bienestar físico y emocional de las personas de acuerdo con sus necesidades. El cuidado tiene un componente material, visible en actividades concretas que pueden ser cuantificables y a las que es posible adjudicarles un valor económico, como lavar ropa, bañar, vestir o alimentar personas dependientes, entre otras. También tiene un componente afectivo o vincular, en la medida en que cuidar supone establecer vínculos emocionales para satisfacer esas necesidades materiales, como brindar cariño, asegurar protección, acompañar, escuchar, entre otros. El cuidado, por tanto, supone establecer un vínculo emocional mutuo entre quien cuida y quien recibe los cuidados (Hochschild, 1983 y 1995). Desde esta perspectiva, cuidar significa la realización de tareas en la vida cotidiana y, simultáneamente, el desarrollo de un vínculo y la gestión de las emociones en los distintos ámbitos donde se realice.

Como muestran los múltiples estudios sobre el tema, las organizaciones sociales del cuidado presentan una distribución desigual que provoca que las responsabilidades del cuidado recaigan en los hogares y en particular en las mujeres. Ello es producto de varios factores que interactúan entre sí, como la persistente división sexual del trabajo, la naturalización de las mujeres como cuidadoras, los escasos desarrollos institucionales de los regímenes de bienestar y las grandes desigualdades económicas (Batthyány, 2020).

Estas configuraciones varían en los diferentes contextos sociohistóricos y espaciales, pero manteniendo su rasgo de fuerte desigualdad de género. De esta forma, el cuidado tiene rostro de mujer y se realiza comúnmente en los hogares sin percibir una remuneración a cambio. La feminización del cuidado se basa en la naturalización de este como una tarea exclusivamente femenina (Araujo Guimarães, Hirata y Sugita, 2011).

En Uruguay, como en el resto de Latinoamérica, la organización social del cuidado se caracteriza por una provisión familiar de los cuidados y por la segmentación en el acceso a servicios y prestaciones públicas y privadas de cuidados (Batthyány y Genta, 2020). 
A partir del año 2010, en Uruguay se inició un proceso pionero de construcción de un Sistema Nacional Integrado de Cuidados (en adelante, SNIC) que busca promover la corresponsabilidad entre familias, Estado, mercado y comunidad, así como la distribución equitativa del cuidado entre varones y mujeres (corresponsabilidad de género). El sistema de cuidados tal cual fue concebido en Uruguay no tiene antecedentes en el mundo en cuanto a la diversidad de poblaciones que integra y a la mirada sistémica o transversal que propone. ${ }^{1}$ El SNIC entiende por cuidados

las acciones que las personas dependientes deben recibir para garantizar su derecho a la atención de las actividades y necesidades básicas de la vida diaria por carecer de autonomía para realizarlas por sí mismas. Es tanto un derecho como una función social que implica la promoción del desarrollo de la autonomía personal, atención y asistencia a las personas dependientes (Ley n. $\left.{ }^{0} 19.353,2015\right)$.

Si bien en la última década el desarrollo de estas políticas ha permitido avanzar hacia la corresponsabilidad social y de género, aún hay una serie de restricciones importantes. En términos generales, existe escasez de servicios públicos y gratuitos de cuidado que cubran la jornada laboral completa para las personas en situación de dependencia. Las políticas de tiempo para cuidar, como las licencias, se han desarrollado solo para los niños pequeños, mantienen un sesgo maternalista y no llegan hasta el año de vida (Perrotta, 2020). Junto a estas dificultades en términos de oferta de servicios y políticas públicas, subsisten representaciones sociales tradicionales sobre el género y el cuidado que asocian a las mujeres y a las familias al cuidado. Como se ha evidenciado, los mandatos de género que vinculan a las mujeres al cuidado directo y a los varones al empleo y al rol de proveedores económicos, así como aquellas valoraciones que identifican que la casa y la familia son los espacios ideales de cuidado, continúan presentes en la sociedad uruguaya (Batthyány y Genta, 2016; Batthyány, Genta y Perrotta, 2014).

Las investigaciones en el país refieren básicamente a contextos urbanos y son casi nulos los trabajos que tienen como objeto de conocimiento especí-

1 El SNIC establece como sujetos de derecho a las personas en situación de dependencia: niños de hasta 12 años, personas con discapacidad y personas mayores de 65 años. La población infantil que el Primer Plan de Cuidados toma como prioritaria es la población de 0 a 3 años. Los niños en este tramo etario representan el 4,6\% de la población que reside en el medio rural disperso o localidades del interior de menos de 5000 habitantes. Por su parte, la población mayor de 65 años representa el 13,6\% de la población rural (elaboración propia basada en la Encuesta Continua de Hogares [ECH] 2018 del Instituto Nacional de Estadística [INE]). Según datos del último Censo de Población (INE, 2011), el 16,4\% de la población rural presenta algún tipo de discapacidad: el $12,1 \%$ al menos una discapacidad leve, el 3,6\% al menos una discapacidad moderada y el 0,6\% al menos una discapacidad severa. En el medio rural disperso las personas con alguna discapacidad son el $14,2 \%$, mientras que en la ruralidad nucleada ascienden al 17,5\%. 
fico los cuidados en el medio rural. Avanzar en estudios sobre la provisión y distribución de los cuidados a nivel macro (Estado, mercado, familias y comunidad) y microsocial (entre varones y mujeres) es central para desentrañar uno de los principales mecanismos de (re)producción de las desigualdades de género en las sociedades rurales actuales y para promover transformaciones en pos de la autonomía de las mujeres.

El presente artículo muestra resultados de una investigación empírica en curso que tiene como objetivo general el conocimiento y la comprensión de la organización social del cuidado en el medio rural y sus pequeñas localidades, desde una perspectiva de género y derechos. ${ }^{2}$ En particular, este artículo se orienta a identificar y discutir las políticas públicas de cuidados implementadas hasta el año 2020 y a analizar las principales trabas para la desfamiliarización y desfeminización del cuidado en los espacios rurales de Uruguay. Para ello se utiliza como base el material empírico recabado en el marco del proyecto, que consiste en entrevistas a actores locales y referentes de espacios de cuidados en pueblos rurales y mujeres asalariadas agropecuarias. ${ }^{3}$

En la primera parte del artículo se presenta una breve revisión de la literatura sobre cuidados y ruralidad. En el segundo apartado se desarrollan los antecedentes en la temática para Uruguay. En el tercero se analiza el sistema de cuidados presente en la ruralidad uruguaya, mostrando sus principales características, las limitantes y los desafíos para la desfamiliarizacion y desfeminización del cuidado. Para cerrar el artículo se presentan las reflexiones finales en función de los hallazgos presentados.

\section{Pensar los cuidados desde la ruralidad}

En el medio rural las desigualdades de género son más visibles, a la vez que tienen mayor impacto que en el medio urbano (Bock, 2004; Díez, 2007; Bock y Shorthall, 2006; OIT, 2018). Las propias características del entorno rural en el que viven ofrecen a las mujeres menores oportunidades para minimizar o reducir los efectos de la desigualdad en sus proyectos vitales (Camarero, 2011).

2 Proyecto "Sistema de cuidados en el medio rural: género e inclusión laboral", Programa Investigación e Innovación para la Inclusión Social, Comisión Sectorial de Investigación Científica (CSIC), Universidad de la República (UDELAR), 2020-2022. Se toma una definición amplia de población rural, entendida como la población residente en el medio rural disperso y localidades de menos de 5000 habitantes (ruralidad nucleada), independiente de su vínculo con la actividad agraria.

3 Entre los meses de febrero y diciembre de 2020 se realizaron 40 entrevistas presenciales a referentes de pueblos rurales; 20 entrevistas virtuales (por Zoom, principalmente) a referentes de espacios de cuidados en marcha o en proceso de implementación; y 1 taller presencial con mujeres asalariadas del agro de los rubros arándano, citricultura, tambo y avícola. Estas actividades se realizaron en Villa del Carmen, Sarandí del Yi, San Gregorio de Polanco, Paso de los Toros, La Paloma, Cuchilla de Peralta, Clara, Centenario, Carlos Reyles, Blanquillo, Arévalo, Tomás Gomensoro, Sequeira, Paso Campamento, Aguas Corrientes, Soca, Rincón de Velázquez, Quebracho, Belén, Villa Constitución, La Charqueaday Puntas de Maciel. 
Como se desarrollará, la literatura académica establece que uno de los principales factores que contribuyen a acrecentar las desigualdades de género en el medio rural tiene que ver con la mayor implicación de las mujeres en las actividades domésticas y de cuidado. En este sentido, como muestran múltiples investigaciones empíricas, en las zonas rurales las mujeres son las encargadas principales de los cuidados (Sampedro, 2009; Camarero, 2009; Cruz-Souza, 2010; Álvarez et al., 2012; Alberti et al., 2014; Minguela y Camacho, 2011; Martín y Rivera, 2018; Rojas, 2018), que en muchos casos deben compatibilizar con sus trabajos remunerados fuera del hogar o en el propio predio en el que residen, en el caso de las productoras familiares, haciendo más pesada su carga global de trabajo.

La feminización del cuidado se debe a una compleja combinación de factores estructurales y culturales que tienen que enfrentar las mujeres en general, así como a una serie de obstáculos que condicionan a las mujeres rurales en particular.

Por una parte, en la ruralidad dispersa o nucleada, el acceso a servicios de cuidados presenta mayores dificultades en función de que la red de cobertura pública y privada es más débil o inexistente (Camarero, 2009). Si bien hay mucha variabilidad en la situación de los espacios rurales (desde zonas despobladas y aisladas a pueblos comunicados y con mayor accesibilidad), en general la oferta de cuidados es insuficiente, puesto que la dispersión poblacional no permite la escala necesaria para la instalación de servicios de cuidados, como muchos otros (Peña y Uribe, 2013). Para suplir esta ausencia en la zona, la población debe trasladarse a otras localidades, lo cual no siempre es posible debido a las distancias, la carencia de transporte público, los tiempos y costos de traslado, el estado de la caminería rural, entre otros. Por eso, en la mayoría de los casos, la población rural queda circunscrita a las posibilidades que le ofrece su entorno cercano, que, al ser muy escasas, obligan a desplegar otras estrategias de cuidado que terminan resolviéndose mayoritariamente en el seno de la familia o con arreglos informales (por ejemplo, los vecinos). Es decir, en la medida en que ni el Estado ni el mercado dan alternativas de cuidado, este pasa a los hogares y, dentro de ellos, a las mujeres.

Otra barrera estructural a la corresponsabilidad en los cuidados tiene que ver con los desequilibrios demográficos que presentan algunas zonas rurales (baja densidad poblacional, masculinización, envejecimiento). La mayor presencia de población envejecida y la menor proporción de mujeres adultas, sumadas a la falta de servicios, aumentan la presión de cuidados sobre las mujeres (Cerri, 2013; Sabaté, 2018). Según Martín y Rivera (2018), las mujeres rurales de la generación soporte padecen la denominada paradoja en el cuidado informal, en la medida en que, por un lado, aumenta la demanda de cuidados (población más envejecida, mayor esperanza de vida de las personas dependientes), mientras que, por el otro, hay un declive en la disponibili- 
dad de personas que cuiden (menos personas en el hogar y en muchos casos con trabajo fuera de este). La relativa escasez de equipamientos y servicios públicos (educativos, sanitarios, culturales, entre otros) es efecto y causa a la vez de esta estructura demográfica (Sampedro, 2008).

Concomitantemente, los factores culturales vinculados a los roles de género tradicionales tienen un papel central en las diferentes modalidades de cuidado desplegadas. En los entornos rurales, la división sexual del trabajo es de corte más conservador que la observada en el medio urbano y, por ende, las representaciones sociales familistas ${ }^{4}$ del cuidado tienden a estar más arraigadas que en el medio urbano (Batthyány, 2013; Kirby y Prolongo, 2013; Alberti et al., 2014; Peña y Uribe, 2013).

En esta línea, Sampedro (2008) señala que el estereotipo tradicional de la mujer centrada en el hogar y la familia es un factor de integración e identidad de la comunidad rural, y las mujeres que se apartan de este modelo enfrentan la desaprobación social. Las dificultades para acceder a dispositivos de cuidado propias del entorno se convierten en un justificativo de domesticidad que es altamente valorado. Para la autora, este vínculo entre domesticidad, feminidad y ruralidad presente en las representaciones sociales explica, en parte, la persistencia de unos roles de género muy tradicionales, que llevan a la baja o nula implicación de los varones en las tareas domésticas y a la resistencia al uso de servicios de cuidado.

En esta misma dirección, Halliday y Little (2001) afirman que las estrategias de cuidado deben analizarse en relación con la disponibilidad de diferentes tipos de servicios, y fundamentalmente en relación con las oportunidades laborales que tienen varones y mujeres en estos espacios y la naturalización de las mujeres como madres y cuidadoras. En la ruralidad, la imagen de la mujer trabajadora continúa rodeada de prejuicios, como el descuido del hogar, la pareja y los hijos, por lo cual se refuerza el rol doméstico de las mujeres y su participación en el mercado laboral se vive en permanente tensión (Fawaz-Yissi y Soto, 2012).

$\mathrm{Si}$ bien en las generaciones más jóvenes se vislumbra un proceso de cambio cultural al cuestionar la naturalización de los roles tradicionales de género, aún persisten y predominan patrones culturales e ideológicos que reproducen las desigualdades en el medio rural (Sabaté y Díaz, 2003; Gatica, 2009; Alberti et al., 2014).

Estos factores, entre otros, se entretejen entre sí y determinan que las estrategias adoptadas por los hogares estén fundamentalmente vinculadas al cuidado informal, teniendo como principal fuente de cuidado de las personas

4 Los términos familismo o familista caracterizan a aquellas representaciones sociales del cuidado que señalan como más deseable que este sea asumido por miembros de la familia. Asimismo, se utilizan para identificar la organización social del cuidado en la cual la responsabilidad se asume mayor o exclusivamente por las familias (Batthyány, Genta y Perrotta, 2014, p. 336). 
dependientes a sus miembros y casi exclusivamente a las mujeres. Son ellas quienes realizan la mayoría del trabajo doméstico y de cuidados como forma de sostener la reproducción de la vida de la familia. Se encargan del cuidado de personas dependientes y de las actividades domésticas (lavar, limpiar, cocinar, realizar mandados y compras, planchar), pero también de actividades de cuidado de animales, labores en la chacra y preparación de cultivos para el autoconsumo. Son actividades que suponen una dedicación completa, donde no hay feriados, ni vacaciones ni jornadas a término (Peña y Uribe, 2013; Artacker, Santillana y Valencia, 2020; Oliveira y Faria, 2018; Herrera, 2016). La brecha de género en el cuidado se refleja en que las mujeres le dedican mucho más tiempo que los varones. ${ }^{5}$ Por ejemplo, un estudio en San Juan (Argentina) muestra que las mujeres destinan diariamente cuatro horas y veinte minutos en promedio a las actividades domésticas, en comparación con la hora que dedican los varones. En cuanto al cuidado, las mujeres le dedican en promedio tres horas, mientras que los varones cuarenta minutos (Kirby y Prolongo, 2013). Este patrón prevalece tanto cuando el varón trabaja remuneradamente como cuando no lo hace (Gatica, 2009).

Estas inequidades en el uso del tiempo son más profundas en el medio rural que en el urbano. Es decir, el tiempo invertido por las mujeres rurales en el cuidado es mayor que el de los varones, pero también que el de las mujeres urbanas (Ortega, 2012; Samman, Presler-Marshall y Jones, 2016; Artacker, Santillana y Valencia, 2020; Wang et al., 2020). Estas desigualdades se agravan cuando las mujeres rurales son pobres (Caro, 2017), indígenas o afrodescendientes (Parada y Butto, 2018).

Las estrategias basadas en el cuidado familiar y femenino conllevan múltiples consecuencias negativas para el desarrollo de la autonomía (económica, política, física) de las mujeres rurales, en tanto estas asumen individualmente el costo del cuidado desde edades tempranas. También presentan menores tasas de participación en el mercado de trabajo y menores oportunidades de acceso a empleos estables y bien remunerados (Ballara y Parada, 2009; Kirby y Prolongo, 2013; De Pablo Valenciano, Capobianco Urdiales y Uribe Toril, 2017; Sánchez, Herrera y Perrotini, 2015). Las mujeres deben adaptar su participación en el mercado laboral en actividades y con jornadas que les permitan conciliar con sus compromisos familiares (Fawaz-Yissi y Soto, 2012). En los casos en que se produce la incorporación de las mujeres al mercado de empleo, ello no supone una redistribución de las tareas de cuidado, sino que las mujeres mantienen su responsabilidad y, por tanto, aumentan su carga global de trabajo (Sifuentes et al., 2018). El tiempo dedicado a

5 La medición del uso del tiempo en el medio rural reporta dificultades adicionales debido a la "invisibilidad" del trabajo que realizan las mujeres, producto de la desvalorización del trabajo doméstico, lo que se refleja tanto en la forma en que se levantan las estadísticas como en la autopercepción que tienen las mujeres de lo que es o no trabajo (Camarero y Oliva, 2004; Chiappe, 2005; Peña y Uribe, 2013). 
los cuidados por parte de las mujeres rurales impone fuertes limitantes a la hora de conciliar la vida laboral con la vida familiar y condiciona la participación laboral de las mujeres y sus modalidades. Las mujeres rurales "disminuyen sus posibilidades de estudiar, trabajar de forma remunerada, heredar propiedades, transitar libremente, cuidar su salud y disponer de tiempo para el ocio y el descanso" (Alberti et al., 2014, p. 397). Tienen una falta sostenida de tiempo personal y de recreación y ocio, tanto durante la semana como en feriados o fines de semana (Sabaté y Díaz, 2003; Gatica, 2009; Oliveira y Faria, 2018). De esta forma, la sorbrecarga de cuidados limita las posibilidades de las mujeres de construir un proyecto de vida independiente de las necesidades de los miembros de sus familias (Herrera, 2017).

Estas inequidades de género se vieron fuertemente reforzadas en el contexto mundial de pandemia por COVID-19. Según Artacker, Santillana y Valencia (2020), las mujeres rurales aumentaron su carga de trabajo no remunerado encargándose aún más de las tareas de cuidado en sus hogares y en la comunidad. La mayor demanda de cuidados para las mujeres supuso un fuerte retroceso en su autonomía económica, provocando su salida del mercado laboral y aumentando la precarización de las condiciones laborales, principalmente en sectores altamente feminizados (salud, educación, servicio doméstico). Para la CEPAL (2021) ello representa un retroceso de más de diez años en la participación de las mujeres latinoamericanas en el mercado laboral. En las zonas rurales, es posible que esta situación se vea marcadamente agravada.

\section{Mujeres rurales y cuidados en Uruguay}

En Uruguay, como se mencionó, las pautas de cuidado se basan en un modelo familístico donde la familia es la principal proveedora. A su vez, en este modelo tradicional, las construcciones de género colocan a las mujeres como principal recurso para los cuidados de niños, adultos mayores y personas con discapacidad. Los antecedentes nacionales sobre las estrategias de cuidado han analizado los hogares urbanos, considerando un contexto de servicios y de movilidad asociado a las ciudades, muy diferente al de los espacios rurales. ${ }^{6}$

Los pocos estudios en la ruralidad indican que predomina también un modelo familístico y femenino de cuidado, más marcado que en los espacios urbanos. Las mujeres rurales son las responsables exclusivas de las tareas de trabajo doméstico y de cuidados cotidianos, las que concilian en muchos casos

$6 \quad$ Las encuestas de uso del tiempo y trabajo no remunerado en el Uruguay, como la Encuesta Nacional de Desarrollo Infantil y Salud (2013 y 2018) del Instituto Nacional de Estadística, son representativas de las localidades urbanas de más de 5000 habitantes, por lo cual no permiten conocer las modalidades predominantes de cuidados en el medio rural. 
con el trabajo productivo (Chiappe, 2008; Peaguda, 1996; Niedworok, 1996; González y Deus, 2010; Batthyány, 2013; Vitelli, 2013; Mascheroni, 2016).

En las explotaciones familiares, la difícil separación entre la unidad productiva y la unidad doméstica hace que las mujeres tengan una doble carga de trabajo que deben conciliar permanentemente. En este sentido, una investigación realizada en productores familiares ganaderos (Batthyány, 2013) muestra que no existe un reparto equilibrado de la responsabilidad sobre el trabajo doméstico y de cuidados: las mujeres realizan el 94\% del trabajo doméstico, el $87 \%$ de las compras y gestiones del hogar y el $70 \%$ de las tareas de cuidado de niños y dependientes. Las mujeres rurales, participen o no del trabajo productivo del predio o en actividades remuneradas fuera de este, son las que se encargan del trabajo no remunerado necesario para la reproducción social y biológica de sus hogares. Estos resultados coinciden con los hallazgos de Bernheim (2018) en establecimientos agropecuarios de tipo familiar y mediano de la ganadería de carne, lechería y horticultura. En todas las actividades del espacio doméstico relevadas, entre ellas el cuidado, la tasa de participación femenina es altamente superior a la masculina.

La mayor carga de responsabilidades familiares con las personas en situación de dependencia, es decir, que requieren apoyos para la vida cotidiana, es acompañada por una escasez de centros de atención para personas dependientes, así como por largas y costosas distancias que se deben recorrer para acceder a los diferentes servicios, como salud y educación, que insumen más tiempo y esfuerzo físico (González y Deus, 2010). Este déficit de los servicios de cuidados en las zonas rurales refuerza aún más el modelo familístico de cuidados.

Estas estrategias basadas en el cuidado familiar y femenino y la falta de un sistema público de cuidados tienen importantes consecuencias en el vínculo con el mercado laboral por parte de las mujeres, las que tienen inserciones más limitadas, precarias e intermitentes. En este sentido, son las mujeres quienes asumen individualmente los costos del cuidado y, por tanto, limitan seriamente su desarrollo profesional y su autonomía económica presente y futura. Mascheroni y Riella (2016) destacan que para las mujeres rurales las actividades de cuidado que recaen sobre ellas restringen el tiempo disponible para asumir actividades laborales fuera del hogar. Salvo excepciones, en los entornos rurales no hay un sistema de cuidado de personas dependientes que permita a las mujeres una inserción laboral de jornada completa. En un contexto que se caracteriza por una demanda reducida de trabajo para las mujeres, el aislamiento y la dispersión, la movilidad diaria es un recurso habitual para el acceso al empleo. Sin embargo, por su mayor implicación en el cuidado de personas dependientes y en el trabajo doméstico asociado a estas actividades, las mujeres ven reducida su movilidad en mayor medida que los varones. Las tareas domésticas propiamente dichas (lavar, limpiar, cocinar, planchar, entre otras) que están a cargo de las mujeres implican mayor tiempo que en el medio urbano, donde 
ciertas facilidades provistas por el propio mercado pueden reducir esta carga. El costo y el tiempo que insume el traslado a otro lugar y la dificultad para dejar a las personas a su cargo en alguna institución que satisfaga la necesidad de cuidado, sumados a los bajos ingresos de los empleos a los que acceden, que no les permiten el pago de servicios privados de cuidados, les hace más difícil la inserción laboral y la búsqueda de oportunidades más allá de su localidad (Mascheroni y Riella, 2016).

En síntesis, los estudios sobre trabajo doméstico y cuidados en la ruralidad uruguaya proporcionan indicios sobre la permanencia de estereotipos de género rígidos, producto de la división sexual del trabajo tradicional entre varones y mujeres. De esta forma, las mujeres son las responsables casi exclusivas del cuidado de personas dependientes. El resultado es que disponen de mucho menos tiempo libre para el trabajo, la formación, el ocio, el descanso y la participación en organizaciones, lo que plantea importantes obstáculos a su autonomía económica, política y física.

\section{Políticas del cuidado en el medio rural}

Desde su creación, el SNIC buscó la universalización de los cuidados y para ello impulsó una descentralización territorial que contemplara las necesidades específicas de cada localidad y contexto. El aterrizaje de estas políticas en el medio rural supuso el desafío adicional de pensar alternativas específicas que atendieran las particularidades de estos espacios y su mayor rezago en cuanto a provisión pública y privada de servicios de cuidados.

En este sentido, es importante remarcar el punto de partida muy diferente del medio rural respecto al urbano. En los contextos rurales, hay una menor mercantilización de los cuidados de personas dependientes, ya que prácticamente no hay oferta privada — la densidad de población hace inviables económicamente los emprendimientos privados-. En cambio, la posibilidad de acceder a servicios mercantiles es mucho más alta en el medio urbano y se encuentra en expansión, principalmente para las clases medias y altas (Batthyány, Genta y Scavino, 2016). En el medio rural, el Estado es el proveedor casi exclusivo de servicios formales de cuidado, pero la oferta existente es escasa y fragmentada: no permite cubrir una jornada laboral completa y muestra falencias en su cobertura en general y en particular a menores de tres años, adultos mayores y personas con discapacidad. Se destacan experiencias puntuales en algunas localidades donde las organizaciones sociales de la zona o grupos de vecinos han implementado algunos dispositivos de atención a la población para suplir la falta de servicios o extender el horario de cobertura de forma de permitir la conciliación laboral-familiar de las mujeres. Ante la falta de servicios privados, públicos o comunitarios, la población rural termina recurriendo a redes familiares o soluciones informales de bajo costo para el cuidado. 
En el medio rural, el SNIC replica algunas de las alternativas desplegadas a nivel nacional, pero fundamentalmente supone el desarrollo y la adaptación de dispositivos particulares pensados para cada zona y con una fuerte participación de la comunidad local en la definición de las necesidades de cuidado y las alternativas viables (diagnósticos participativos). En general, la política pública avanzó en tres ejes:

a. ampliación de políticas universales existentes de atención a la infancia, aumentando su cobertura y mejorando su calidad: Centros de Atención a la Infancia y a la Familia (CAIF), jardines de infantes, Centros de Atención a la Primera Infancia (CAPI);

b. despliegue en territorios rurales de servicios de cuidado creados a nivel nacional: Casas Comunitarias de Cuidados (CCC), becas de inclusión socioeducativa, Programa Teleasistencia en Casa, Programa Asistentes Personales, licencias paternales;

c. implementación y apoyo de servicios innovadores específicos para el medio rural: espacios multimodales de cuidados (EMC) y asistentes comunitarios.

En la última línea se trabajó en la construcción participativa de dispositivos adecuados a las necesidades de cada localidad. Desde el SNIC se realizaron talleres con la población local a partir de los cuales se identificaron necesidades y opciones posibles que culminaron en la propuesta de espacios multimodales de cuidados y la ampliación de una experiencia de asistentes comunitarios. ${ }^{7}$

De esta forma, la oferta de cuidados en el medio rural combina servicios institucionales (escuelas, CAPI, CAIF, CCC, espacios multimodales de cuidados), políticas de tiempo (licencias parentales) y prestaciones monetarias (becas de inclusión socioeducativa). En el anexo incluido al final de este artículo se sintetizan las características de los dispositivos de cuidado infantil $\mathrm{y}$ de personas dependientes que funcionan en el medio rural.

\section{Limitantes de las políticas del cuidado en el medio rural}

En este apartado se realiza una reflexión crítica sobre la implementación de las políticas de cuidado en la ruralidad uruguaya que muestra la importancia de considerar el entrelazamiento de factores estructurales y culturales para entender las estrategias de cuidado presentes y el alcance de las políticas públicas del cuidado.

7 En el marco del proyecto "Municipios que cuidan. Pueblos que cuidan", impulsado por la Secretaría Nacional de Cuidados (SNC) y la Oficina de Planeamiento y Presupuesto (OPP), se elaboraron 17 propuestas en localidades de menos de 3000 habitantes (Sequeira y Tomás Gomensoro en Artigas, Aguas Corrientes, Santa Rosa, Santa Lucía y Soca en Canelones, Villa del Carmen, Blanquillo y La Paloma en Durazno, Belén, Villa Constitución y Colonia Lavalleja en Salto, La Charqueada y Vergara en Treinta y Tres, Quebracho en Paysandú). 


\section{a. Limitantes estructurales}

De las entrevistas y el relevamiento realizados surge claramente que la disponibilidad de servicios públicos, privados y comunitarios de cuidados es muy restrictiva. La oferta de los instrumentos que tiene disponible el SNIC es muy escasa y no llega a todos los territorios. Entre ellos, se observan notables diferencias en cuanto a la posibilidad de servicios de cuidado (asociada en parte a la cantidad de población, la cercanía a centros urbanos, la accesibilidad a las localidades, la demanda local, entre otras). Los espacios disponibles son fundamentalmente públicos y la mercantilización del cuidado es muy baja.

Sumado a esta carencia, hay un desequilibrio importante en la oferta de cuidados según grupos. La población infantil es la que tiene mayor cobertura, pero esta es aún muy deficiente: la mayoría de los servicios de cuidado son de cuatro horas, por lo que no cubren una jornada laboral completa; en algunos casos se destaca que no hay cupos para atender a todos los niños y prácticamente no hay opciones para menores de 3 años.

En cuanto a los adultos mayores en situación de dependencia, es para esta población donde se visualiza la mayor necesidad de cuidados. En los espacios rurales, salvo contadas excepciones, no hay centros de larga estadía ni centros diurnos públicos, y prácticamente no existen servicios privados. En algunas zonas se cuenta con clubes de recreación o sociales para personas mayores autoválidas, pero no hay dispositivos extendidos para quienes están en situación de dependencia o con alguna discapacidad. Por tanto, la atención domiciliaria o en espacios de cuidado de personas dependientes es uno de los temas que genera mayor preocupación y demanda en la población. En cambio, la demanda de servicios para las personas con algún grado de discapacidad no surge claramente en la población, a pesar de que la carencia de servicios para este grupo es aún mayor.

Lo otro que pasa acá, que es semirrural, que tenés que viajar, es el adulo mayor que no tiene un lugar. Por ejemplo, no hay un hogar de ancianos, no tenemos esas cosas acá. Cuando ya llegan a una altura el abuelo se va del pueblo porque a veces las familias no los pueden tener porque trabajan y nadie los puede cuidar, y terminan en un residencial, pero siempre fuera de Aguas Corrientes (Entrevista a referente).

La demanda referida a la población con discapacidad surgió en aquellos municipios que tienen más población con discapacidad que el promedio, donde el tema supera la media. Donde no supera la media está asumido que lo cuida la madre o la hermana. No tienen posibilidades de moverse ni en el pueblo. Realmente es la población que queda más excluida y menos visible para demandar; si no demandan sus padres quedan invisibilizados y dentro de sus casas (Entrevista a referente). 
Otra limitante refiere a las dificultades para asegurar la calidad de los servicios que se ofrecen. En los entornos rurales, los recursos humanos y técnicos no son suficientes y deben suplirse con personas de otras localidades, lo cual es una tarea compleja - e imposible en muchos casos- por los problemas de movilidad y conectividad en función del estado de la caminería, la lejanía de los centros poblados y la baja frecuencia de las líneas de transporte público. Esto se ve agravado por situaciones climatológicas que provocan el aislamiento de ciertas localidades.

[Principales obstáculos] En primer lugar adquirir las ayudas técnicas, como sabrás, en el entorno rural no tenemos cómo acceder a un mercado variado de ayudas técnicas. Como por ejemplo si necesitás un andador para una persona, porque no todas las personas necesitan el mismo andador, el mismo bastón, la misma silla de ruedas. Tenemos que viajar a Montevideo para hacer ese estudio. Y después, obviamente los recursos humanos, y profesionalizarlos en lo que es el área. Porque no contamos con eso acá en la localidad, siempre tienen que venir de afuera o tienen que trasladarse (Entrevista a referente).

La baja densidad de población actúa como un limitante para el traslado de los servicios a las pequeñas localidades, pero, por otro lado, permite universalizar la atención una vez que se instala el dispositivo de cuidado, por ejemplo, en el caso de los niños cuando se instala una escuela, jardín o casa comunitaria. La llegada de la institucionalidad al territorio permitió, además, generar otros movimientos, como la detección de situaciones de violencia basada en género, problemas de nutrición y otras problemáticas.

Junto a la carencia de servicios, otra limitante que se presenta a las políticas públicas del cuidado es la dificultad para universalizar dispositivos pensados a nivel nacional.

Uno de los grandes desafíos, y también fortaleza del SNIC, es la necesidad de pensar alternativas que se adapten a cada uno de los territorios rurales en los cuales se asienta. Tempranamente se vio la imposibilidad de replicar la totalidad de los instrumentos pensados para espacios urbanos, así como también se visualizó que las diferencias entre territorios (densidad de población, cercanía a centros urbanos con servicios, accesibilidad y transporte, características productivas de la zona, entre otros) requerirían opciones diversas y adaptadas a cada uno.

Esta diversificación y adecuación supuso la interinstitucionalidad y el involucramiento de los actores del territorio. Para sacar adelante la propuesta de cuidados fue necesaria la articulación entre diferentes instituciones públicas, tanto para la elaboración de las propuestas como para su financiamiento. El diálogo interinstitucional generó tensiones entre las perspectivas de las diferentes instituciones involucradas, principalmente en el caso de los servicios de primera infancia, vinculadas a los debates sobre la calidad del servicio. 
Asimismo, se requirió un proceso de involucramiento de los actores políticos de diferentes niveles (nacional, departamental, municipal) y de movilización de la comunidad en torno a alternativas de cuidado posibles para el lugar donde se iban a desarrollar.

Estos aspectos pusieron trabas a la extensión de experiencias de cuidados debido al tiempo y los acuerdos políticos que se requieren para su implementación. En los casos en los que no hubo apoyo de los gobiernos departamentales o municipales, no se iniciaba el proceso. Pero, por otra parte, en los casos en los que se avanzó se logró proponer servicios de cuidados adaptados a las necesidades de la ruralidad y de cada territorio. No obstante, las experiencias que pudieron instrumentarse son muy limitadas y su sostenibilidad está cuestionada por los cambios en la orientación de la política de cuidados del nuevo gobierno. ${ }^{8}$

Adicionalmente, el SNIC debe enfrentar varias dificultades para la extensión de algunos servicios o prestaciones. Tal es el caso del Programa de Asistentes Personales, por el requerimiento de formación que exige a quienes cuidan. ${ }^{9}$ Para suplir esta limitante, la llegada de este servicio fue a través de la excepcionalidad de la prestación, permitiendo que se contrate a personas que no tengan la capacitación específica (aunque sí manifiesten un compromiso de adquirirla), en el entendido de que dicha formación no ha llegado a la totalidad de las localidades. Otra limitante señalada es la falta de disponibilidad de personas interesadas en desarrollar la tarea de asistentes personales en ciertos territorios, lo que dificulta su implementación. Respecto al Programa de Teleasistencia, inicialmente el servicio requería una conexión por medio de una línea de teléfono fijo, lo cual era una barrera para las personas residentes en la ruralidad debido a la escasa cobertura de este servicio.

\section{b. Limitantes culturales}

Las representaciones sociales sobre el género y el cuidado condicionan las estrategias de cuidado desplegadas por los hogares rurales. En las entrevistas se reproducen estereotipos tradicionales de género que determinan las significaciones que se construyen en torno a los arreglos familiares para el cuidado y la compatibilización trabajo-familia por parte de las mujeres.

8 De los proyectos derivados de "Municipios que cuidan. Pueblos que cuidan" (SNC-OPP) comenzaron efectivamente dos (Quebracho te Cuida y Vergara te Cuida). El resto continúa en proceso de implementación y, por cambios en la política nacional de cuidados del nuevo gobierno, se han enlentecido los procesos o se han restringido los fondos para su continuidad (tal es el caso de asistentes comunitarios de Vergara te Cuida).

9 Para la habilitación como asistente personal se debe aprobar un curso básico de capacitación certificado por el Ministerio de Educación y Cultura. La realización de estos cursos en pequeñas localidades fue muy limitada y, por tanto, no se logró formar suficientes recursos humanos locales. 
En este sentido, las definiciones sobre los roles femeninos naturalizan a la mujer como madre y cuidadora. Las mujeres desarrollan, así, la mayor parte de las tareas reproductivas y de cuidado y su trabajo productivo es visto como una "ayuda" para el hogar o el establecimiento familiar. Desde este punto de vista, la imagen de la mujer rural que trabaja fuera de su casa mantiene en muchos casos una valoración negativa.

La mayoría de las mujeres no trabaja, esa es la realidad. Y no sé si están con muchos deseos de trabajar. Es más cultural. Yo me llevo bien con todo el mundo y la realidad de la gente es "Paulita, andá a dormir, andá con tu hija, no seas tan mala madre". Pasás a ser mala madre porque trabajás mucho (Entrevista a referente).

La dificultad de las mujeres rurales para armar un proyecto personal propio más allá de la maternidad y la familia, y las escasas oportunidades que ofrece el entorno en el que viven refuerzan su rol de cuidadoras, al que dedican su tiempo y mayor esfuerzo.

Hay toda una identidad y un uso del tiempo que se desarma si yo saco el factor cuidados, hay un cambio, ¿y yo qué hago con ese cambio en un lugar donde yo no tengo posibilidades ni de salir a caminar?, porque quizás tenga esa posibilidad, pero si salgo, ¿qué van a pensar los demás que no estoy en mi casa? Porque todo eso pasa, ¿no? (Entrevista a referente).

Tenés que pedirle por favor a las madres para que los lleven, no los quieren llevar (Entrevista a referente).

Bueno, yo creo que atraer a la gente es uno de los desafíos, porque nada, eso: "No hay nada cerca, y en realidad ya me organicé para cuidarlo todo el día yo, y prefiero no salir a trabajar". O sea, entre ir a trabajar y quedarme cuidando a mi hijo, a veces las elecciones son "me quedo cuidando a mi hijo" porque no hay nada cerca. Entonces, creo que uno de los principales desafíos es poder atraer a la gente y decirles: "Sí, podés salir. Podés salir a trabajar, a estudiar o a hacer lo que quieras, o a prestarte atención o a leerte un libro debajo de la palmera". Ahí va, creo que uno de los principales desafíos es poder generar esa confianza de la población en el cuidado, en que sientan que es un servicio para ellos y no de control (Entrevista a referente).

A su vez, las características asignadas a las mujeres, como sensibilidad, paciencia y afectividad, hacen que sean percibidas como más adecuadas a la hora de brindar cuidados, independientemente, en general, de la preparación personal que puedan tener. 
[Las mujeres] Y tienen más paciencia. Gran parte de las cosas pasan por la paciencia (Taller de mujeres).

Lo emocional juega un rol muy importante. Yo emocionalmente me sentía bien, como que no sentía el cansancio que traía porque yo sabía que en los momentos que yo estaba es cuando él [padre] se sentía tranquilo, se sentía bien, se sentía cuidado porque estaba yo. Podés tener la mejor capacitación del mundo, pero tenés que estar también capacitada emocionalmente (Taller de mujeres).

La mujer es más sensible. Del hecho de parir en adelante. Eso de ser madre te da una sensibilidad. Te da un plus (Taller de mujeres).

Esta naturalización de la mujer como cuidadora supone cierta tensión entre lo que se atribuye al instinto y lo que es o puede ser aprendido. Se percibe que las mujeres tienen una condición innata para el cuidado ("es algo que se trae") y que los varones, en algunos casos y sobre todo los más jóvenes, lo pueden aprender.

La mujer lo trae pero el hombre lo adquiere. Lo venimos trayendo desde la cuna. Pero como hay mujeres que no les importa nada (Taller de mujeres).

Las demandas de cuidado se resuelven principalmente en los hogares, en parte por la falta de servicios de cuidado, pero fundamentalmente por los diferentes mandatos de género y representaciones acerca de qué es el "buen cuidado". En este sentido, el cuidado ideal es considerado aquel que proveen las familias y en particular las mujeres, tanto en el cuidado de niños más pequeños como de los progenitores y personas con discapacidad. Esta centralidad de la familia conlleva la preferencia por brindar una atención individualizada y afectiva.

El ingreso de los niños a instituciones se entiende que es adecuado a partir de los 2 o 3 años. En el caso de los bebés o niños menores de 3 años, lo ideal se considera el cuidado en el seno del hogar o por personas de la familia: "Porque uno ve la parte emocional más que nada, el vínculo emocional, el vínculo de sangre" (Taller de mujeres). A partir de esta edad, es más aceptada la asistencia de niños a centros educativos y, por tanto, compartir la responsabilidad del cuidado. En cuanto a los adultos mayores y las personas con discapacidad, no hay resistencias al uso de espacios de cuidado de horario parcial y es una demanda recurrente en la ruralidad. En cambio, hay una desconfianza hacia la institucionalización plena, especialmente en geriátricos, y una preferencia por el cuidado brindado en casa y por la familia, que se considera que satisface mejor las necesidades emocionales y afectivas de las personas. 
¿De qué me sirve a mí meter a mi vieja en un geriátrico? Sé que va a estar atendida, sé que va a tener un médico al lado, sé que va a tener una enfermera al lado, pero no va a estar en su entorno familiar que es lo que a ella la hace sentir bien. Porque a veces están bien de salud, pero los metés en esos lugares y terminan enfermándose porque no están bien emocionalmente (Taller de mujeres).

Estos mandatos de género que asocian a las mujeres al cuidado directo actúan como un freno a las políticas que pretenden impulsar cambios en el sentido de desfamiliarizar y desfeminizar el cuidado, en tanto generan resistencias de la población para el uso de los servicios disponibles, así como actúan generando una baja demanda de políticas de cuidado.

En los talleres la demanda más sobresaliente era vinculada a las personas mayores. Los servicios de cuidados no son pensados como respuesta a las necesidades de cuidados de la infancia, pues se prefieren redes familiares a través del cuidado de familiares directos. Está muy instalado que es la hermana mayor quien cuida, quien abandona su trayectoria educativa para asumir responsabilidades de cuidado. Ahora, una vez que instalás el servicio se usa y es altamente valorado (Entrevista a referente).

Cabe resaltar que la baja demanda de servicios de cuidados de la primera infancia es construida. En contextos en donde la naturalización de los roles de las mujeres es tan amplia y la estructura de oportunidades tan restringida, se justifica que no se perciba la necesidad de más centros o de mayor cobertura u horarios compatibles con la jornada laboral. Se establece así un círculo vicioso en la demanda/oferta de cuidados. Por un lado, la falta de demanda visible de servicios (centros de cuidado o extensión de horas de estos) genera que esos territorios no sean considerados para su instalación. A su vez, la falta de oferta de servicios provoca que no sean percibidos como una opción de cuidado que sustituya a la familia y que, por tanto, no sean espacios apropiados, valorados y demandados por la población. Por ende, es necesario analizar al mismo tiempo la oferta de servicios y prestaciones disponibles con la demanda que surge en la localidad y entender esta demanda desde la lógica de las mujeres en esos territorios. Es decir, la demanda de cuidados se construye a partir de lo que se identifica como una necesidad. Si el rol de las mujeres está asociado a los cuidados y naturalizado, probablemente no aparezca la necesidad ni la demanda de servicios que "sustituyan" el cuidado de los niños durante algunas horas del día para conciliar con su vida laboral, educativa o recreativa. 


\section{Reflexiones finales}

En este artículo se aborda uno de los principales nudos de las desigualdades de género en los territorios rurales, que tiene que ver con el cuidado. En los últimos años se produjo un avance sustantivo al colocar los cuidados en el centro de agenda pública y política, y al proponer miradas desde la ruralidad con enfoque de género que promueven la desfamiliarización y la desfeminización del cuidado.

Pese a ello, aún se está muy lejos de consolidar un sistema de cuidados en el medio rural y más bien lo que se observa es un conjunto limitado de acciones mediante las que se han puesto a prueba diferentes dispositivos buscando su adaptación a la ruralidad. Los hallazgos de la investigación sugieren que persisten severas limitaciones para la universalización de los cuidados en el medio rural, las que se explican en parte por el entrecruzamiento de trabas estructurales y culturales. Las limitantes estructurales están vinculadas a las propias características del contexto (aislamiento, estructura poblacional, lejanía), a la falta de servicios disponibles y a la dificultad de adaptación de instrumentos para cada territorio rural. Pero las mayores restricciones se encuentran asociadas a factores culturales que refieren a las prácticas y las representaciones tradicionales del cuidado. En el medio rural persisten mandatos de género y de cuidado que asocian el "buen cuidado" con aquel brindado por la familia y, en particular, por las mujeres. La naturalización de los cuidados familiares y femeninos se manifiesta en el menor uso y demanda de servicios de cuidado. La combinación de estos factores estructurales y culturales provoca que el cuidado recaiga fundamentalmente en las mujeres, limitando sus posibilidades de construir con mayor autonomía sus proyectos de vida. Las escasas alternativas de servicios de cuidados promueven más la familiarización y feminización, y, a su vez, esta preferencia de cuidados familiares invisibiliza la demanda de servicios.

En este sentido, si bien el cambio institucional y el aumento de los servicios formales de cuidados son importantes para generar transformaciones en las estrategias de cuidado de los hogares rurales, no son suficientes para promover su corresponsabildad. Se requiere un importantísimo esfuerzo de sensibilización en género que problematice y genere transformaciones en las representaciones y valoraciones sobre el cuidado que legitiman y naturalizan que sea la madre, la hermana o la abuela la que cuida y que sobrecarga a las mujeres rurales limitando su propia autonomía. Desde el SNIC, en diferentes pueblos rurales se realizaron talleres y exposiciones sobre corresponsabilidad en los cuidados, pero estos deben ser mucho más extendidos para que efectivamente se puedan deconstruir estereotipos muy arraigados en la población rural y en las propias mujeres rurales.

Asimismo, la forma particular en que se entrelazan y articulan los factores estructurales y culturales en el medio rural confirma la pertinencia de 
pensar desde estos territorios acciones y medidas tendientes a la corresponsabilidad social y de género en los cuidados. Ciertamente, existe un retraso en el avance del movimiento feminista en el campo que enlentece los procesos de debate en la temática. No obstante, en los últimos años las organizaciones de mujeres rurales han colocado el tema de cuidados en la agenda (INMUJERES-MIDES, 2015), con una visión hegemónica más vinculada a las necesidades de salud en el caso de las productoras y asociada al ejercicio de los derechos laborales — entre ellos los vinculados a la maternidad-y la conciliación de la vida laboral-familiar en el caso de las asalariadas.

En las mujeres rurales se solapan diferentes desigualdades de género, clase y territorio que limitan sus oportunidades y dificultan el logro de su autonomía. En este contexto, el cuidado es una dimensión clave de la equidad de género. Sin dudas, las modificaciones en las relaciones de género y en las estrategias de cuidado son importantes fuerzas impulsoras de cambio social y de reducción de las inequidades de género en la ruralidad. Por tanto, avanzar en la corresponsabilidad de los cuidados entre varones y mujeres, y entre Estado, mercado, familias y comunidades es fundamental para la reducción de las desigualdades en los territorios rurales. La ruta a trazar en esta dirección debe estar pensada desde la ruralidad y con la participación de las mujeres que viven o trabajan en estos espacios. Asimismo, se requiere una permanente reflexión crítica y problematización desde una perspectiva de género sobre las diferentes políticas y opciones de cuidado que se implementen desde el Estado, el mercado y la comunidad, ya que no necesariamente promueven la equidad de género y la autonomía femenina. ${ }^{10}$

Finalmente, es fundamental generar mayor conocimiento sobre los mecanismos concretos que hacen que en el medio rural se reproduzcan más tradicionalmente las relaciones de género, tanto en las representaciones sociales como en las prácticas, para poder avanzar en políticas que permitan reducir las desigualdades y los sesgos de género, que, como se mencionó, no solo están presentes en las familias, sino también en el Estado, el mercado y las comunidades.

10 Por ejemplo, Van Rompaey y Scavino (2020) llaman la atención sobre que el Programa de Asistentes Personales del SNIC, al invisibilizar la dimensión vincular y emocional del trabajo de cuidados en domicilios, producto de la naturalización de un saber hacer por parte de las mujeres, perpetúa condiciones de desvalorización y desprotección de las tareas de cuidados, y de esta forma reproduce las desigualdades de género. 


\section{Referencias bibliográficas}

Alberti-Manzanares, P.; M. Zavala-Hernández; B. Salcido-Ramos y N. RealLuna (2014). Género, economía del cuidado y pago del trabajo doméstico rural en Jilotepec, Estado de México. Agricultura, Sociedad y Desarrollo, 11(3), pp. 379-400.

Álvarez Sánchez, Y.; R. D. Díaz Mateus y J. Enrique Saiz Vélez (2012). Empresas de familia rurales, relaciones de género, relaciones de poder. Caso Lenguazaque, Cundinamarca. Criterio Libre, 10(17), pp. 53-72.

Araujo Guimarães, N.; H. Sumiko Hirata y K. Sugita (2011). Cuidado e cuidadoras: o trabalho de care no brasil, frança e japão. Sociología \& Antropología, 1(1), pp. 151-180.

Artacker, T.; A. Santillana y B. Valencia (2020). En el centro la vida: mujeres rurales tejiendo cuidado y movilización. CLACSO [en línea]. Disponible en: <https://www.clacso.org/en-el-centro-la-vida-mujeres-rurales-tejiendocuidado-y-movilizacion/> [acceso: 25/01/2021].

Ballara, M. y S. Parada (2009). El empleo de las mujeres rurales: lo que dicen las cifras. Roma: FAO-CEPAL.

Batthyány, K. (2013). Uso del tiempo y trabajo no remunerado: división sexual del trabajo y contratos de género. Un estudio de caso en el medio rural familiar. En: D. Piñeiro, J. Cardeillac y R. Vitelli (coords.). Relaciones de género en el medio rural uruguayo: inequidades a la intemperie. Montevideo: Facultad de Ciencias Sociales, Universidad de la República, pp. 81-106.

Batthyány, K. (2020). Recorridos latinoamericanos de los cuidados. En: K. Batthyány (coord.). Miradas latinoamericanas a los cuidados. Buenos Aires-México: CLACSO-Siglo XXI, pp. 11-52.

Batthyány, K. y N. Genta (2020). Uruguay: avances y desafíos en la investigación y las políticas públicas del cuidado. En: N. Araujo Guimarães y H. Hirata (comps.). El cuidado en América Latina: mirando los casos de Argentina, Brasil, Chile, Colombia y Uruguay. Ciudad Autónoma de Buenos Aires: Fundación Medifé Edita, pp. 129-257.

Batthyány, K; N. Genta y V. Perrotta (2014). Las representaciones sociales del cuidado infantil desde una perspectiva de género. Principales resultados de la Encuesta Nacional sobre Representaciones Sociales del Cuidado. Papers 2014, 99(3), pp. 335-354. 
Batthyány, K.; N. Genta y V. Perrotta (2017). El aporte de las familias y las mujeres a los cuidados no remunerados en salud en Uruguay. Revista Estudos Feministas, 25(1), pp. 187-213.

Batthyány, K.; N. Genta y S. Scavino (2016). Análisis de género de las estrategias de cuidado infantil en Uruguay. Revista Cadernos da Pesquisa, 47(163), pp. 292-319.

Bernheim, R. (2018). Estudio sobre estrategias de adaptación al cambio climático y género para el Plan Nacional de Adaptación a la Variabilidad y Cambio Climático para el Sector Agropecuario (PNA-Agro). Montevideo: FAO-PNUD-MGAP.

Bock, B. (2004). It still matters where you live: Rural women's employment throughout Europe. En: H. Buller y K. Hoggart (eds.). Women in the European countryside, Aldershot, Ashgate, pp. 14-41.

Bock, B. y S. Shorthall (2006). Rural Gender Relations. Issues and Case Studies. CAB International Publishing. London.

Camarero, L. (coord.) (2009). La población rural de España. De los desequilibrios a la sostenibilidad social. Barcelona: Fundación La Caixa.

Camarero, L. (2011). Agricultoras rurales: una profesión desigual. En: E. Muñiz Espada (coord.). Un marco jurídico para un medio rural sostenible. Ministerio de Medio Ambiente y Medio Rural y Marino, pp. 311-324.

Camarero, L. y J. Oliva (2004). Las trabajadoras invisibles de las áreas rurales: un ejercicio estadístico de estimación. EMPIRIA. Revista de Metodología de Ciencias Sociales, 7, pp. 159-182.

Caro Molina, P. (2017). Desigualdad y transgresión en mujeres rurales chilenas: Lecturas desde la interseccionalidad, género y feminismo. Psicoperspectivas. Individuo y Sociedad, 16(2), pp. 125-137.

CEPAL (2020). La autonomía económica de las mujeres en la recuperación sostenible y con igualdad. Informe especial COVID-19 n.o 9 [en línea]. Santiago de Chile: CEPAL. Disponible en: < https://repositorio.cepal.org/ bitstream/handle/11362/46633/5/S2000740_es.pdf $>$ [acceso: 10 de febrero de 2021].

Cerri, C. (2013). El impacto de los servicios públicos de cuidado a mayores en una zona rural de Extremadura. Gazeta de Antropología [en línea], 29(2). DOI: 10.30827/Digibug.28499. 
Chiappe, M. (2005). La situación de las mujeres rurales en la agricultura familiar de cinco países de América Latina. Montevideo: ALOP.

Chiappe, M. (2008). El enfoque de género y la situación de las mujeres rurales. En: M. Chiappe, M. Carámbula y E. Fernández (eds.). El campo uruguayo: una mirada desde la sociología rural. Montevideo: Facultad de Agronomía, Universidad de la República, pp. 241-258.

Cruz-Souza, F. (2010). Las desigualdades de género en el trabajo dentro de las fincas agrarias familiares. Soberanía Alimentaria, Biodiversidad y Culturas [en línea], 2. Disponible en: <https://revistasoberaniaalimentaria.wordpress.com/2010/08/23/las-desigualdades-de-genero-en-el-trabajo-dentrode-las-fincas-agrarias-familiares/> [acceso 07/05/2021].

De Pablo Valenciano, J.; M. Capobianco Urdiales y J. Uribe Toril (2017). Vulnerabilidad laboral de la mujer rural latinoamericana. Nóesis. Revista de Ciencias Sociales y Humanidades, 26(52), pp. 130-151.

Díez Bedmar, M. (2007). Las mujeres en el mundo rural. Reflexiones generales y contextualización. Sumuntán: anuario de estudios sobre Sierra Mágina, 24, pp. 67-78.

Domínguez, L. y F. Brown (2013). Diferencias de género en la elección del sitio de trabajo en un contexto de crisis. Revista CEPAL, 111, pp. 83-102.

Elizalde-San Miguel, B. (2017). El cuidado informal en las zonas rurales. Nuevas formas, nuevos actores. Social and Education History, 6(2), pp. 168-195.

Fawaz-Yissi, J. y C. Rodríguez-Garcés (2013). Mujeres rurales y trabajo en Chile central. Actitudes, factores y significaciones. Cuadernos de Desarrollo Rural, 10(72), pp. 47-68.

Fawaz-Yissi, J. y P. Soto (2012). Mujer, trabajo y familia. Tensiones, rupturas y continuidades en sectores rurales de Chile central. Revista de Estudios de Género La Ventana, 35, pp. 218-254.

Gatica Godoy, C. (2009). El uso del tiempo de la mujer rural: entre la familia y el trabajo. Realidades actuales y representaciones de la mujer rural, de la comuna de El Carmen, Provincia de Ñuble, Chile. Tesis para optar al grado académico de Magíster en Familia mención en Intervención Familiar. Universidad del Bío-Bío, Chile.

González Perrett, D. y A. Deus (2010). Análisis legislativo: derechos de las mujeres rurales y equidad de género. Proyecto: "Uruguay, hacia el país de la 
equidad: formación de agentes de desarrollo social desde un enfoque de género en apoyo a la formulación e implementación del Plan Nacional de Igualdad de Oportunidades y Derechos (PIODNA)". Montevideo: FAOINMujeres/Ministerio de Desarrollo Social.

Halliday, J. y J. Little (2001). Amongst Women: Exploring the Reality of Rural Childcare. Sociología Ruralis, 41(4), pp. 25-49.

Herrera, K. M. (2016). Da Invisibilidade ao Reconhecimento: mulheres rurais, trabalho produtivo, doméstico e de care. Política \& Sociedade, 15, pp. 208233.

Herrera, K. M. (2017). Repensando o valor social do trabalho das mulheres rurais. Ponencia presentada en el Seminário Internacional Fazendo Gênero 11 \& 13th. Women's Worlds Congress (Anais Eletrônicos), Florianópolis.

Hochschild, A. R. (1983). The Managed Heart. Commercialization of human feeling. California: University of California Press.

Hochschild, A. R. (1995). The culture of politics, Traditional, Postmodern, Coldmodern, and Warm-modern ideals of care. Social Politics, 2(3), pp. 332346.

INE (2011). Censo de Población, Hogares y Viviendas. Montevideo: Instituto Nacional de Estadística (INE).

INE (2018). Encuesta Continua de Hogares (ECH). Montevideo: Instituto Nacional de Estadística (INE).

INMUJERES-MIDES (2015). Encuentro Nacional Voces de Mujeres Organizadas: construyen una agenda nacional. Documento sintesis. Montevideo: Instituto Nacional de las Mujeres, Ministerio de Desarrollo Social.

Kirby, A. S. y S. Prolongo (2013). Problemáticas sociales de mujeres de una zona semi-rural, Departamento 9 de Julio-Provincia San Juan. Revista Dos Puntas, V(7), pp. 131-148.

Little, J. (1987). Gender relations in rural areas: the importance of women's domestic role. Journal of Rural Studies, 3(4), pp. 335-342.

Martín Gómez, A. y J. Rivera Navarro (2018). Feminización, cuidados y generación soporte cambios en las estrategias de las atenciones a mayores dependientes en el medio rural. Prisma Social: Revista de Investigación Social, 21, pp. 219-242. 
Mascheroni, P. (2016). Diagnóstico prospectivo en brechas de género y su impacto en el desarrollo. Mujeres rurales: Trabajo y acceso a recursos productivos. Montevideo: Dirección de Planificación, Oficina de Planeamiento y Presupuesto.

Mascheroni, P. y A. Riella (2016). La vulnerabilidad laboral de las mujeres en áreas rurales. Reflexiones sobre el caso uruguayo. Revista de Ciencias Sociales, 29(39), pp. 57-72.

Maya Frades, V. (ed.) (2008). Mujeres rurales: estudios multidisciplinares. Salamanca: Ediciones Universidad de Salamanca.

Minguela Recover, M. y J. Camacho Ballesta (2011). Dependencia: Cuidados formales e informales en Andalucía. Diferencias entre el ámbito rural y urbano. Ponencia presentada en el III Congreso Anual de la REPS (Red ESpañola de Política Social). Universidad Pública de Navarra, en Pamplona.

Morales, M. (2016). Consultoría para el diseño metodológico para la identificación de servicios informales de cuidado para la primera infancia. Apoyo a la Secretaría Nacional de Cuidados. Sistema Nacional Integrado de Cuidados. Montevideo: Banco Interamericano de Desarrollo.

Niedworok, N. (1986). La mujer rural: familia y trabajo en el Uruguay. En: N. Filgueira (comp.). La mujer en el Uruguay: ayer y hoy. Montevideo: GRECMU-EBO, pp. 224-239.

Oliveira Teixeira, M. y N. Faria (2018). Empoderamento económico das mulheres no Brasil pela valorização do trabalho doméstico e do cuidado. San Pablo: Oxfam Brasil.

OIT (2018). Rural Women at Work: Bridging the gaps [en línea]. s. 1.: OIT. Disponible en: <https://www.ilo.org/wcmsp5/groups/public/---ed_protect/--protrav/---ilo_aids/documents/publication/wcms_619691.pdf $>$ [acceso: $8 / 10 / 2020]$.

OPP (2020). Sistematización: Fortalecimiento de la llegada del SNIC a pequeñas localidades. Municipios que cuidan. Pueblos que cuidan. Montevideo: Oficina de Planeamiento y Presupuesto.

Ortega Ponce, L. (2012). Las relaciones de género entre la población rural del Ecuador, Guatemala y México. Santiago de Chile: CEPAL.

Pacheco, E. y N. Florez (2014). Entre lo rural y urbano: tiempo y desigualdades de género. En: B. García y E. Pacheco (coords.). Uso de tiempo y trabajo no 
remunerado en México. Ciudad de México: El Colegio de México, ONUMujeres, INMUJERES, pp. 263-324.

Parada, S. y A. Butto (2018). Avances y desafios de las mujeres rurales en el marco de la estrategia de género del plan SAN-CELAC 2025. Santiago de Chile: Organización de las Naciones Unidas para la Alimentación y la Agricultura.

Peaguda, M. C. (1996). Las mujeres productoras de alimentos en Uruguay: diagnóstico y políticas. San José de Costa Rica: IICA/BID.

Peña, X. y C. Uribe (2013). Economía del cuidado: valoración y visibilización del trabajo no remunerado. Colombia: Centro de Estudios sobre Desarrollo Económico, Universidad de los Andes.

Perrotta, V. (2020). Género y políticas de cuidado en Uruguay: ¿avanzando en una relación virtuosa? En: K. Batthyány (coord.). Miradas latinoamericanas a los cuidados. Buenos Aires-México: CLACSO-Siglo XXI, pp. 187-231.

Rojas, M. (2018). Mujeres jóvenes rurales, sus estrategias laborales, y la economía del cuidado en la provincia de San Juan, Argentina. Revista Latinoamericana de Antropología del Trabajo, 2(4), pp. 1-21.

Sabaté Martínez, A. (2018). ¿Qué significa ser mujer en zonas rurales? Boletín ECOS, 42, pp. 1-7.

Sabaté, A. y M. A. Díaz (2003). Mujeres y desarrollo rural: la conciliación de tiempos de vida y de trabajo. Serie Geográfica, 11, pp. 141-162.

Samman, E.; E. Presler-Marshall y N. Jones (2016). Women's workMothers, children and the global childcare crisis. Londres: Overseas Development Institute.

Sampedro; R. (2008). Conciliación de la vida familiar y laboral en el medio rural: género, trabajo invisible e "idilio rural". En: V. Maya Frades (ed.). Mujeres rurales: estudios multidisciplinares de género. Salamanca: Ediciones Universidad de Salamanca, pp. 81-93.

Sánchez, A.; A. Herrera e I. Perrotini (2015). La participación laboral femenina y el uso del tiempo en el cuidado del hogar en México. Contaduría y Administración, 60(3), pp. 651-662.

Sifuentes Ocegueda, E. L.; K. Y. Rivera Flores y A. T. Sifuentes Ocegueda (2018). Tiempos de vida de las mujeres en el medio rural. Trabajos de cuidados y opciones productivas en Nayarit, México. Revista de investigación y divulgación sobre los estudios de género, 25, pp. 105-138. 
SNIC-MIDES (2019). Implementación de las Casas Comunitarias de Cuidados. Una mirada de los servicios, las personas que cuidan, las familias, los niños y las niñas, y la comunidad. Montevideo: SNIC, Ministerio de Desarrollo Social.

Tronto, J. (1993). Moral Bounderies. A Political Argument for an Ethic of Care. Londres: Routledge.

Tronto, J. (2010). Creating caring institutions: politics, plurality, and purpose. Ethics and Social Welfare, 4(2), pp. 158-171.

Tronto, J. (2013). Caring Democracy: Markets, Equality and Justice, Nueva York: NYU Press.

Tronto, J. (2017). La democracia del cuidado como antídoto frente al neoliberalismo. En: C. Domínguez Alcón, H. Kohlen, J. Tronto. El futuro del cuidado. Comprensión de la ética del cuidado y práctica enfermera. Barcelona: Ediciones San Juan de Dios, pp. 19-36.

Uruguay, Poder Legislativo (2015). Ley n.o 19.353 Creación del Sistema Nacional Integrado de Cuidados (SNIC), 8 de diciembre.

Van Rompaey, E. y S. Scavino (2020). La “trampa de género" en la invisibilización de la dimensión emocional en el trabajo remunerado de cuidados. Ciudadanías. Revista De Políticas Sociales Urbanas, 7, pp. 1-29.

Vitelli, R. (2013). Un examen de las relaciones de género en el medio rural. En: D. Piñeiro, J. Cardeillac y R. Vitelli (coords.). Relaciones de género en el medio rural uruguayo: inequidades a la intemperie. Montevideo: Facultad de Ciencias Sociales, Universidad de la República, pp. 49-61.

Wang, Y.; J. Li; N. Zhang; L. Ding; Y. Feng; X. Tang; L. Sun y C. Zhou (2020). Urban-Rural Disparities in Informal Care Intensity of Adult Daughters and Daughters-in-Law for Elderly Parents from 1993-2015: Evidence from a National Study in China. Social Indicators Research [en línea]. DOI: 10.1007/s11205-020-02280-4. 
Anexo

\section{Cuadro 1. Servicios de cuidados para el medio rural}

\begin{tabular}{|c|c|c|}
\hline Programa & $\begin{array}{l}\text { Condición de } \\
\text { acceso }\end{array}$ & Características \\
\hline \multicolumn{3}{|r|}{ Primera infancia } \\
\hline $\begin{array}{l}\text { Centros de } \\
\text { educación } \\
\text { inicial } \\
\text { privados }\end{array}$ & 0 a 5 años & $\begin{array}{l}\text { Flexibilidad horaria } \\
\text { Servicio pago }\end{array}$ \\
\hline $\begin{array}{l}\text { Escuela } \\
\text { pública }\end{array}$ & $\begin{array}{l}\text { A partir de } 3 \\
\text { años }\end{array}$ & $\begin{array}{l}\text { Escuela rural } 5 \text { horas } \\
\text { Escuela suburbana y urbana } 4 \text { horas }\end{array}$ \\
\hline Jardín público & 3 a 5 años & $\begin{array}{c}4 \text { horas en su mayoría } \\
? \text { horas y media tiempo completo }\end{array}$ \\
\hline CAIF & 0 a 3 años & $\begin{array}{c}4 \text { horas en su mayoría } \\
\text { Política intersectorial de alianza entre organismos públicos, } \\
\text { gobiernos municipales y asociaciones civiles. La prestación } \\
\text { del servicio y la gestión de los recursos brindados por } \\
\text { organismos públicos para los CAIF quedan a cargo de } \\
\text { asociaciones civiles. }\end{array}$ \\
\hline CAPI & $\begin{array}{l}3 \text { meses a } 3 \\
\text { años }\end{array}$ & Horario flexible. \\
\hline $\begin{array}{l}\text { Casas } \\
\text { Comunitarias } \\
\text { de Cuidados }\end{array}$ & $\begin{array}{l}45 \text { días hasta } \\
12 \text { meses (con } \\
\text { posibilidad } \\
\text { de hasta } 36 \\
\text { meses] }\end{array}$ & $\begin{array}{l}\text { Hasta } 40 \text { horas semanales. } \\
\text { Existen dos modalidades: a) en domicilio: un cuidador que } \\
\text { atiende a dos niños en su propio hogar; b) espacio físico } \\
\text { comunitario: dos o tres cuidadores atienden hasta quince } \\
\text { niños. } \\
\text { Se articula con los CAIF cercanos para recibir soporte } \\
\text { técnico. }\end{array}$ \\
\hline Internado rural & $\begin{array}{l}\text { A partir de } 3 \\
\text { años }\end{array}$ & Escuela con internado de lunes a viernes. \\
\hline $\begin{array}{l}\text { BIS [Becas } \\
\text { de Inclusión } \\
\text { Socioeducativa }\end{array}$ & 0 a 2 años & $\begin{array}{c}\text { El objetivo de estas becas es costear el servicio de cuidados } \\
\text { y educación a niños y niñas de } 0 \text { a } 2 \text { años pertenecientes } \\
\text { a familias vulnerables cuando no hay oferta de servicios } \\
\text { públicos en el territorio. }\end{array}$ \\
\hline $\begin{array}{l}\text { Licencia } \\
\text { maternal, } \\
\text { paternal y } \\
\text { medio horario } \\
\text { para cuidados }\end{array}$ & Hasta 6 meses & $\begin{array}{l}\text { Trabajadores privados, independientes y monotributistas. } \\
\text { Ausencia total y reducción horaria hasta } 4 \text { horas diarias. }\end{array}$ \\
\hline
\end{tabular}




\begin{tabular}{|c|c|c|}
\hline Programa & $\begin{array}{c}\text { Condición de } \\
\text { acceso }\end{array}$ & Características \\
\hline \multicolumn{3}{|c|}{ Personas en situación de dependencia } \\
\hline $\begin{array}{l}\text { Espacio } \\
\text { Multimodal } \\
\text { de Cuidados } \\
\text { [EMC] }\end{array}$ & $\begin{array}{l}\text { Personas en } \\
\text { situación de } \\
\text { dependencia }\end{array}$ & $\begin{array}{l}\text { Horario flexible (no funciona diariamente, sino que se } \\
\text { establece una grilla de actividades). } \\
\text { Son espacios de atención y promoción de la autonomía, } \\
\text { diseñados a partir de las problemáticas y los recursos } \\
\text { locales. Cada propuesta es singular y se diseña en función de } \\
\text { las necesidades y demandas de los territorios. } \\
\text { Se orientan a la atención de personas en situación de } \\
\text { dependencia desarrollando actividades recreativas, de } \\
\text { socialización, de estimulación física y cognitiva para la } \\
\text { promoción de la autonomía y el retraso de la dependencia, así } \\
\text { como para el apoyo y asesoramiento a familiares y personas } \\
\text { cuidadoras de quienes asisten al EMC. } \\
\text { Sus líneas de acción tienen que ver con: la atención } \\
\text { sociosanitaria preventiva y de estimulación para las } \\
\text { actividades de la vida diaria, el apoyo a familiares } \\
\text { y personas cuidadoras de las personas usuarias, la } \\
\text { integración comunitaria. }\end{array}$ \\
\hline $\begin{array}{l}\text { Asistentes } \\
\text { personales }\end{array}$ & $\begin{array}{l}\text { Hasta } 29 \text { años } \\
\text { y mayores de } \\
80 \text { años en } \\
\text { situación de } \\
\text { dependencia } \\
\text { severa }\end{array}$ & $\begin{array}{c}80 \text { horas mensuales. } \\
\text { Subsidio total o parcial. } \\
\text { Prestación económica destinada a la contratación de } \\
\text { asistente a domicilio para el cuidado y la asistencia } \\
\text { personal para las actividades de la vida diaria (alimentarse, } \\
\text { higienizarse, vestirse, movilizarse, trabajo, estudio y } \\
\text { recreación, entre otras]. }\end{array}$ \\
\hline $\begin{array}{l}\text { Asistentes } \\
\text { comunitarios }\end{array}$ & $\begin{array}{l}\text { Personas } \\
\text { adultas en } \\
\text { situación de } \\
\text { dependencia } \\
\text { leve o moderada } \\
\text { que viven solas } \\
\end{array}$ & $\begin{array}{l}\text { Atención en tareas instrumentales de la vida cotidiana } \\
\text { (elaboración de alimentos, aseo personal, limpieza del } \\
\text { hogar, compra de medicamentos, entre otros] y refacciones } \\
\text { en el hogar para adaptarlo a las necesidades y movilidad } \\
\text { de las personas (rampas, modificaciones en el baño, en la } \\
\text { cocina). }\end{array}$ \\
\hline Teleasistencia & $\begin{array}{l}\text { Mayores de } 70 \\
\text { años con grado } \\
\text { de dependencia } \\
\text { leve o moderada }\end{array}$ & $\begin{array}{l}\text { Subsidio total o parcial. } \\
\text { El servicio funciona a través de una pulsera o collar que } \\
\text { activa una conexión, a través de una línea de teléfono fijo, } \\
\text { que permite que ante cualquier incidente puedan avisar a } \\
\text { su familia, vecinos o referentes médicos. }\end{array}$ \\
\hline
\end{tabular}

\begin{tabular}{lcc}
\hline & Hijos con & Trabajadores privados. \\
Liscapacidad. & (Hijos con discapacidad) 10 días anuales para controles \\
especiales & $\begin{array}{c}\text { Familiares con } \\
\text { discapacidad } \\
\text { o enfermedad } \\
\text { terminal a cargo }\end{array}$ & (Familiares con discapacidad o enfermedad terminal a \\
& cargo) 96 horas anuales. \\
\hline
\end{tabular}

Fuente: Batthyány y Genta (2020) y adaptación propia al medio rural.

\section{Contribución de autoría}

Este trabajo fue realizado en su totalidad por Paola Mascheroni Laport. ${ }^{11}$

11 La autora agradece la lectura atenta y las sugerencias de quienes evaluaron el artículo. 